imminent. Careful attention to the history and examination should enable one to readily differentiate appendicitis from psoas abscess, morbus coxarius, renal and biliary colic, tubal and ovarian disease, intussusception, carcinoma of the colon and cecum, typhoid fever and floating kidney. Peri-nephritic abscess occurring on the right side might readily deceive one as to its origin. The mistake, however, would be of no moment, for the indications in each would be practically the same. When pain is produced in the right iliac fossa by pressure on the left side of the abdomen we should be suspicious of appendicitis.

To sum up the diagnostic symptoms upon which we must rely to recognize appendicitis 1 mention: 1, pain; 2 , nausea and vomiting; 3 , tenderness and muscular resistance in the right iliac fossa; 4, palpation, tumefaction, fluctuation; 5 , septic manifestations.

\section{OF WHAT USE IS SURGICAL TREATMENT IN OVARIAN NEUROSES?}

Read before the Nebraska State Medical Society, at Lincoln, Neb. Mry 19, 1894.

BY A. F. JONAS, M.D.

PROFESSOR OF CLINICAL SURGERY IN THE OMAHA MEDICAL COLLEGE; SURGEON TO OMAHA DEACONESS HOSPITAL, ETC. OMAHA, NEB.

The term neurosıs, as commonly used by the profession, has an indefinite meaning. It may mean a pain, severe or slight, of a "neuralgic character." It is often applied to irregular functional disturbances, as for example, the gastro-intestinal functions, which may be perfect to-day, less so to-morrow, violently disturbed the next day, and normal the fourth, without any assignable cause. Neurosis may mean emotional manifestations, for example, irritability of temper, explosions of unprovoked grief, attacks of epileptiform convulsions, such psychic disturbances as melancholia and mani. acal conditions. Ecstasy, delirium, hallucinations and trance have been called by that convenient term. It is often applied to that indefinite symptom-complex, known as neurasthenia, or more popularly called hysteria. In short, no matter what the train of symptoms may have been, when it seemed impossible to find structural changes due to inflammatory processes, acute or chronic, or when it could not be shown that there existed an atypical cell proliferation in the form of a neoplasm, or other new formation, it has been the custom to speak of it as neurosis, or a neurotic condition. When there has been observed pain, aberration of glandular or organic function; psychic or mental disturbances, convulsive manifestations, without discernible organic structural changes, that much abused term, neurosis, has been found of great con. venience.

Under the modern system of specialism, when each organ has its special custodian, whose sole duty seems to be to stand as a guardian angel over that which he has selected and adopted, it seems clear to each specialist that this long train of neurotic symptoms, are nothing more nor less than reflexes (another term used with so much delight, and no more understood than the other), beginning in that particular organ which happens to be the pet of a given specialist. There is such a strong tendency for every specialist to attempt to look into the human body, through his own little speculum.

The ophthalmologist, if he can find nothing visible by external inspection or ophthalmoscopic examination. discovers slight errors of refraction or accommodation. and in that, a reflex neurosis which explains that headache, the disinclination for work or pleasure. The otologist is sure to find the origin for vertigo and fretfulness in the ear.

The rhinologist is positive that the dyspnea and its accompanying train of symptoms, originate in the deflected septum, spurs, thickened turbinated tissue and post-nasal adenoids.

The lung and heart specialist is inclined to ignore what the aforesaid authorities have diagnosticated, for he hears through his stethoscope sounds that explain all.

The gastro-intestinal authority finds matters very plain. He removes the stomach contents after giving a test meal, and with reagents and microscope, and possibly, if he be well advanced and properly equipped, he may employ the gastroscope, and the gyromele. $\mathrm{He}$ indicates to you as plainly as the writing on the wall, what is at the bottom of the trouble.

The orificial specialist is positive, beyond all powers of argument, that all neuroses, and even appendicites, begin in the external orifices.

The gynecologists, and nearly one-half of the physicians of our cities are professed gynecologists, never fail to trace an ache, or pain to the pelvic orguns. The uterus and its adnexa are centers, which, to their minds, control every function in a woman's body. Every ache and pain is a neurosis, and when located in a region remote from the pelvis, is a reflex condition. Under the head of neurosis, according to our enterprising and pushing gynecologist, is included a wide range of disorders, and I fear, a multitude of sins.

When we view this vast subject from the standpoint of the true gynecologist (not the six weeks' policlinic fledgeling, but I mean the earnest, honest, broad minded, careful, painstaking, scientific gynecologist, whose specialty is the culmination of a wide enperience as a general practitioner, and whose skill as a specialist is the outcome of a long and large experience at the bedside), we soon find that we must eliminate the larger portion of nervous manifestations, from our consideration, as diseases originating strictly from the organs of generation which are frequently denominated as ovarian neuroses.

Under the caption of ovarian neuroses, we may include all such affections as are characterized by primary pain or other disturbances, referable to the ovarian regions, unattended by inflammation or any ascertainable structural lesion.

If we adhere strictly to this definition, we will find that the vast majority of the gynecologic cases that come to us, are of a neurotic character. The pelvic distress and hypersthesia, local or diffused, is in a large portion of cases, unaccompanied by structural change. Nearly all these cases complain of distress involving, one, and in some cases, nearly every vital organ in their bodies.

For convenience of description, in our discussion we may adopt the classification of Baldy: 1, neurasthenia; 2, hystero-epilepsy; 3 , psychoses-insanity.

The more modern, more esthetic, and possibly more scientific appellation, neurasthenia, has largely displaced the term hysteria. The latter has become to be regarded as a reflection upon the mental balance of the sufferer, and is often means a forfeiture of svmpathy on the part of her friends. But to designate the long and variable train of nervous and mental 
disorders, as neurasthenia makes her an object of solicitude and the tenderest care from the side of her friends, and stamps the doctor, in the estimation of all concerned, as a skillful diagnostician and a profound physician. While hysteria and neurasthenia are described under separate heads, still when we study their symptomatology we find that both have much in common. We find in hysteria (Webber) "an almost innumerable variety of symptoms, which may be analyzed in a general way into signs of increase, diminution, or perversion of various nervous functions. It is commonly classed as a functional nervous disease, and has no recognizable patholic anatomy. Postmortem investigation gives no clew to the morbid process."

For neurasthenia (James J. Putnam) we find "certain states of the nervous system of which the anatomic basis is unknown, but which is characterized on the one hand by lack of vigor, efficiency and endurance, atfecting usually a large number of nervous functions, and on the other hand, by signs of active derangement, which in part seem to occur as positive symptoms, and in part are due to failure of the mutual control which the different parts of the nervous system afford each other in health."

When we make a critical analysis of the cases that come to us for treatment, we find that nearly twothirds of them belong under the above headings. Their subjective symptoms are innumerable, their objective signs, aside from feebleness and palor due usually to too close confinement and inactivity, are few. Among the endless subjective symptoms, a few usually predominate and are referred to some special organ, and in these progressive days, when pelvic organs have met with such terrible onslaught and have been so persistently directed to the pelvis, the poor sufferer being a woman, naturally thinks of those organs which make her such. She seeks comfort and advice from the specialist, and he being ambitious and anxious to distinguish himself, and being imbued with the idea that the culmination of all skill is in blood, seldom fails to find something in the pelvis, that, to his mind, solves the entire condition and offers him opportunity for sanguinary achievements. If he does not find a laceration of cervix uteri or perineum which need repair, it is a metritis, that must undergo the ordeal of curettement, and if not that, a thicken. ing in one or both broad ligaments, which means chronic salpingo-ovaritis, and if he be an up-to-date specialist, he can invariably diagnose adhesions; or he finds adherent flexions and versions. The diagnosis having been completed a grave prognosis made, an operation is urged as the only means of cure, and often the only possible means of saving life, for blood he craves, and nothing short of blood will appease his craving. The frightened patient gives her consent. Elaborate preparations are made; a half dozen or more colleagues are invited to witness the marvelous feat. Theoperator clad in spotless gown, in his sterilized hand he holds aloft a gleaming, keen-edged knife. He calls time: with a sweep or two of the knife the peritoneal cavity is open; two fingers are rapidly thrust into the sacred recesses of the pelvic cavity; an unoffending ovary and tube, a marvel of perfect anatomic form, is drawn forth and instantly pronounced cystic; it is tied off by a specially devised method, cut away and the pedicle dropped. The same procedure is followed on the other side. To vary the condition and to give proof of his wonderful knowledge of morbid structure, he unhesitatingly pronounces the second ovary (which really does not differ from the first) to be scirrhotic. Abdominal wound closed by a new method, dressing applied and the time-keeper announces the startling information, "seven minutes!" A triumph of modern surgery and individual dexterity! Patient is put to bed, recovery uneventful, discharged, cured on the fourteenth day. Cured, did you say? By that you mean that her neurasthenic manifestations have vanished. Did you ever see one of these chronic sufferers cured in fourteen days? When Lawson Tait only a few years ago published his remarkable tables, of a hundred or more cases in a table, and we found that 98 out of 100 cases were discharged cured, you and $I$, in our verdant simplicity imagined that when he said cured, it meant to be relieved from all pain and distress, and the departed bloom of youth had returned. And when we found that our cases had not been cured in six months or a year, and some were even worse, it slowly dawned upon us, that "cured" in published statistics, meant a survival of the surgical ordeal only. When we examined the appendages removed by the aforesaid seven minutes operation, we found that what we had been taught to be normal, unruptured Graafian follicles had suddenly, according to the aforesaid operator, become cysts. The old sites of the ruptured follicles had become evidences of a "fibrous condition," which was proof positive of scirrhosis. The Fallopian tubes were open at both ends and otherwise so perfect as to delight the teacher of anatomy who is in search of normal specimens to demonstrate to his class. One well-known surgeon, after he had removed the uterine appendages, found that what the textbooks describe as sites of newly ruptured Graafian follicles, an ulceration of the ovaries. Who has not seen several pairs of such ovaries with open Fallopian tnbes, with beautifully branching fimbriæ, exhibited before medical societies, as the result of a half-day's labor. You and I have no hesitancy in pronouncing them to be normal, and we ask why were they taken out? The answer can always be anticipated. "Why, she had ovarian neuralgia:" the "cystic" and "scirrhotic" condition caused reflex neuroses!

We have often wondered why operators in comparatively small towns have such a large proportion of pus tubes among their abdominal cases. Recently we had some light thrown on this point by an operator, who, in a country district where specific infection is a rarity, removed in ten minutes uterine appendages identically like those described in the foregoing sevenminute operation. The operator carefully squeezed the Fallopian tube from its fimbriated toward its uterine end, and succeeded in pressing out of it a very few drops of fluid that had a very slight opaque appearance; instantly the diagnosis of pyosalpinx was made. The microscope was superfluous with a surgeon who possessed such microscopic vision.

If we could examine all the specimens removed from cases in the many reported "series" where the mortality is small we would find a large proportion to be those of absolutely normal uterine appendages. Have you taken pains to follow the subsequent history of these cases? You will find that a large proportion, the majority of them, are not benefited. Many have been made worse. Why? If these cases had been carefully investigated, the conclusion would have been forced upon the operator that the local pain was only a local expression of a general nervous condition. In these cases we sometimes have persistent pain in the 
stomach, liver, heart, lungs, head, arms and legs. Why not extirpate or amputate one or more of these occasionally. But the resourceful gynecologist promptly decides that the pain in these various organs are of a reflex nature, which take their origin in the womb or ovaries. Poor maligned organs, "more sinned against than sinning!"

A few years ago I had occasion to do a successful Morton's operation in a case of metatarsalgia in the left foot of a young married woman. The uterus had been faithfully painted with silver nitrate solution twice a week for several months by a "gynecologist," under the supposition that the pain in the foot and calf of the leg was a uterine reflex.

About a year or more ago I was visited by an unmarried lady, who had suffered for several years from general nervous debility, probably due to over mental strain and deficient physical activity. She had some pelvic pain, somewhat exaggerated during the menstrual period; she had undergone much local th treatment of the form termed "uterine tinkering" by Lawson Tait. She was referred to me for operation because drugs, which had been given chiefly in the form of high potencies, $1 \times 500,000$, had not cured her. On bimanual examination the pelvic organs, which could be unusully well palpated, were found to be absolutely normal. Her chief complaint was gastro-intestinal disorders, obstinate habitual constipation. These had been regarded as due to reflexes. She was advised to return, with a written opinion, to her physician (who had given her no treatment but had referred her to me). She failed to follow my advice; she sought another operator, who promptly removed her ovaries and tubes, collected a large fee and discharged her cured. A few weeks ago she appeared in my office again. A nervous, fretful, emotional, broken down woman. No, hardly a woman, for that which distinguished her sex had been taken from her, without any compensation for her loss. If her tubes and ovaries had undergone structural changes, as the result of disease, she was unsexed already, and to have removed them would have given her health, but to remove her normal uterine adnexa the surgeon is directly responsible for the aggravated neurosis. As she sat in the chair bemoaning her fate and denouncing her surgeon, she cried: "If I had only followed your advice! What shall I do? What shall I do?" I have seen many such cases, and I am sure other doctors have seen similar cases that have been operated on. My personal experience in abdominal work began when pelvic surgery, aside from the removal of large ovarian cysts, was in its infancy. Shortly after the field had been opened by Beatty, Tait and Hegar, for the removal of diseased uterine adnexa. We all of us looked to these men for guid. ance. While their lists of cases were long ones, the information they conveyed was imperfect and meagre and referred only to the immediate results of the operation. Little or nothing could be learned regarding the ultimate outcome. We were compelled to learn much by our own experience, we had no precedents established as we have today. In my anxiety to relieve suffering I frequently operated on cases belonging to the class under discussion, but the ultimate outcome was usually so unsatisfactory that with accumulating experience with each succeeding year I refused to operate on cases that I would have advised surgical procedure in the preceding.

I unhestatingly declare that all cases in which no structural changes in the pelvic organs can be made out, should not be subjected to operation, and in cases where under mistaken diagnosis the abdomen is opened and the appendages found to be normal they should not be removed, no matter what the local subjective symptoms. The experience of such men as Gill Wylie, Boldt, Howard Kelley, Charles Noble, Baldy and many others, has shown that no benefit can be expected, but the cases are often made worse.

Trachelorraphy.-An operation which in popularity stands next before salpingo-ovarectomy is trachelorraphy. A minor operation which every tyro can do, and illustrates the ready credulty of the average patient. It brings the operator as large a fee as the average abdominal section, and has established the reputation of many a man as a "fine surgeon," who would shrink from an ordinary amputation.

Every laceration, however slight, must be repaired, upon the assertion that nerve endings are adherent to the cicatrix, which if they have not already produced, soon will cause a neurosis and innumerable reflexes. This operation, when done according to the indications laid down by Emmet, is useful, but as usually done is useless in four-fifths of the cases, as proven by the results.

Hystero-epilepsy.-According to Charcot and his pupils, hystero-epilepsy "is distinctly a symptom of hysteria, and in no way confounded with epilepsy, when it assumes its typical form." . . "The seizure is usually preceded by a change in disposition, perhaps for some days, and more directly by an aura, abdominal or epigastric, which affords the patient time to seek a position of safety. The attack proper is divided into four periods: 1 , the epileptoid; 2 , the period of contortions and great movements; 3 . the period of emotional attitudes; 4 , the period of delirium" (G. L. Walton). "During the intervals between the attacks the patient suffers from incomplete hysteric hemianesthesia, ovarian hyperesthesia and other hysteric symptoms . . . The aura, which is in the nature of a globus hystericus, seems to proceed from the affected ovary. The patient shrieks and falls insensible, and tonic spasms result; this is quickly followed by clonic spasms and these are succeeded by muscular relaxation, stertorous respiration and coma. The second stage (phase des grands movements) consists of movements which are rhythmical or disordered, and which are sometimes violent and ludicrous. The next stage (phase des attitudes passionelle) is marked by a rapid succession of attitudes and gestures which suggest the term phase of lubricity. Hallucinations of terror, sorrow, joy, etc., follow. The paroxysms may recur frequently and may be provoked by slight pressure upon the ovarian region on certain hyperesthetic spots; sudden and firm compression of the ovary will sometimes arrest a paroxysm" (Foster).

Charcot and his followers believed that this affection had its origin in the womb and its appendages. This view seemed confirmed by the observation that the attacks often became worse during the menstrual period. When abdominal section became a comparatively safe procedure, surgeons did not hesitate to drag forth and remove tubes and ovaries that were often models of anatomic perfection. Occasionally a case was benefited and sometimes cured. These were reported with all the extravagance of which the English language is capable; and many more were reported before sufficient time had elapsed to note if 
the case had even been temporarily benefited. One of the blots of this progressive period in medicine is the premature report of cases.

But what became of the fifty that were not benefited and not reported, but were permitted to wend their miserable way, unpublished and uncured? Hence those of us of more limited opportunities and less experience were led into the error of holding up to our distracted patients a light of hope that burned a short while, then flickered feebly and went out. Have you ever experienced the despair that follows hope, the darkness that succeeds a dazzling light?

My own experience comprises eight cases. When we study these cases we find, in all, the attacks were aggravated during the menstrual period. In seven the attacks occurred between the periods. In one only luring the period. In six the attacks could be brought on by compression of one or both ovaries. In the one case where the attacks occurred during the period only, a complete cure has been effected. In one case, that of a married lady, mother of eight children, the attacks sometimes numbered two or three during an hour. After five years, I was informed that she seldom has more than one convulsion during twenty-four hours, "but that they are very much milder," We see she is not cured. The chief benefit that has been derived is that she can not propagate any more of her kind. In the remaining six cases we imagined for a time that they were improved, but gradually relapsed into their former condition.

When we take into consideration that some of these cases recover spontaneously, we wonder whether those which are seemingly benefited by an operation might not have recovered without one. My own limited experience is not encouraging. On consultation of recent literature I find the results of those operators of large experience is no more encouraging, and I find that the weight of opinion is in favor of surgery only in such cases where the attacks are confined entirely to the menstrual period. In all other cases it is worse than useless to operate.

Even the insane woman has not escaped the enterprising abdominal surgeon. $\mathrm{He}$ studied his cases profoundly and selected carefully such in whom the mental disturbance seemed worse during the menstrual period. They were often reported improved immediately after operation, but we have been kept in the dark in reference to the ultimate outcome. My own experience has taught me that it is an exceed. ingly hazardous procedure to operate on man or woman who has exhibited unusual mental "peculiarities." It has been my misfortune to have had four cases of post-operative insanity. The immediate recovery in all the cases was uneventful. Convalescence had practically been completed when mental disturbance appeared. Without any evidence to the contrary, I feel that we are not justified in doing salpingo-ovarectomy on the insane women, except for organic changes, no matter how aggravated her condition during the period, if she is not perfectly sane during the inter-menstrual time. In cases where insanity occurred only during the flow we might expect recovery after inducing a premature menopause by operative interference. You ask why in the face of this evidence do men continue to mutilate women for functional disorders? We may answer:

1. Inexperience and mistaken diagnosis. No man should begin pelvic work until after many years of general practice, until he has served a long appren- $/$ better health. ticeship with an experienced diagnostician and operator, so that he may avoid the pitfalls into which those of us have fallen when there were no precedents to guide us. The opportunity for acquiring diagnostic skill in our large medical centers is almost unlimited, but it requires time and patience to acquire it. The great trouble about our post-graduate teaching is, that somehow, although the opportunities for becoming proficient in diagnosis are abundant, the student returns to his chosen field with his head full of technique and not enough of diagnostic landmarks.

2. The desire to do many abdominal sections and to show a low mortality ratio in his reports. At this time nothing is more tiring and time-robbing in our society meetings than to report a long series of abdominal sections, each case differing from every other in the list. A surgeon who aims to do many laparotomies always does many useless ones. Such a surgeon takes his own reputation solely into account, irrespective of what benefits his patients may derive. His policy is a short-sighted one. His selfishness predominates in all his work. His many needless operations will in the end cost him his reputation, for which he has so zealously and short-sightedly labored.

3. The prospects of a good fee. The man who is prompted chiefly by a fee (and we know of such) is unworthy of the confidence of his patients and his fellow practitioners. He should be relegated to that oblivion from which he came.

4. The pleadings of the patient. Who can sit unmoved when the poor suffer who has exhausted many methods of treatment without relief appeals to you as a surgeon. I have often found that it required more courage to refuse an operation than to do one. Surgeons should lay sentiment aside at such a time and look the stern facts in the face and decide accordingly.

5. The fear of losing your patient, that she may go to another surgeon. Have the courage of your convictions, state them frankly and plainly and in the end you will be the gainer; subsequent patients will place confidence in your opinion.

We have endeavored to point out what must not be done for these cases. And now more than ever, the great question confronts us. What can we do. for these unhappy beings?

Experience has shown us that the surgery of the present is a failure. We must seek in other directions and for other means which may offer a hope of relief. We have seen that the majority of these cases with functional disturbances are inactive physically and mentally; they are anemic, have muscular atony, poor circulation, gastro-intestinal distress, especially obstinate constipation. It has been my practice for years to insist on some form of physical exercise. If the case is too feeble to do this alone begin with the aid of an assistant, who aids in the various passive and active movements, followed by massage. Later, various forms of gymnasties; then riding, walking in the open air. But the difficulty has been the patient soon ceases to be interested and she returns to her old condition. Of late I have succeeded better. I have prescribed that fascinating and popular modern innovation, the bicycle, one of the most useful machines invented in the last century. In nearly every case the patient has forgotten her medicine and her doctor, and goes wheeling merrily along, with new interest, new plans for life and happiness and 
In this dissertation, it has been my aim to call attention to some of the abuses in our profession. If I have been severe in some of my statements they have been prompted by motives for the improvement of surgery. I have not spared myself. I have frankly spoken of my failures in the hope that my small contributions might assist us in the future to avoid a useless method and starch along new lines. As broad and progressive surgeons we must become, first of all, diagnosticians, not along special and narrow lines, but must be willing to give the time to study and learn the nature of all diseases to which the body is heir, and all possible means for their proper recognition. The gynecologist, especially, should have a most thorough knowledge of neurology, gastro-intes. tinal diseases and affections of the urinary tract. Unless we are thus prepared we will be blunderers, groping mostly in the dark, and our therapeutics will be solely experimental. I am confident, however, that even now we have at our command the means which can and will remove from our profession the stigma of an inexact science. Much has been done for the cure of woman's ills in the last decade. We have only begun to find means for the relief from suffering in her who is the better, nobler and kinder half of humanity. She who is man's inspiration, his joy and his hope.

\section{EXTRA-UTERINE PREGNANCY.}

Read before the State Medical Association of Missouri, May 19, 1896. BY T. F. PREWITT, M.D.

Professor of Principles and Practice of Surgery and Clinical Surgery. Missouri Medical College; Surgeon to St. John's Hospital and Infirmary, etc., St. Isouis, Mo.

In fulfilling the functions for which the sex was created, no accident more dreadful can befall a woman than that of extra-uterine pregnancy. She may have reason to suspect that pregnancy has occurred, but in that fact she finds nothing to excite apprehension of the future. On the contrary it may kindle in her bosom the fondest aspirations of motherhood; the instincts of maternity may arouse in her heart the tenderest emotions and visions of that supreme happiness which fill the soul of the mother, when, having passed the period of probation, she clasps her firstborn to her breast with rapture. Should she already have been a mother, there is nothing different from past experiences and she has no dread of the future. Soon the inevitable catastrophe comes, it may be with the suddenness of the lightning's flash; an agony of pain, collapse and death. It may be all so sudden and violent that no chance of rescue is offered. Yet this is not always so, not even perhaps in a large proportion of cases. The clinical history and certain significant symptoms often serve to warn the practitioner, and woe to his patient if they are not quickly heeded and promptly acted upon. He is confronted with a grave responsibility and if he fails to grasp the situation or misinterprets the symptoms, a life is sacrificed.

Six cases have fallen under my care. Of these, two died and four recovered. Not the least instructive of these are the two fatal cases, both of which I believe would have recovered had I not followed the teachings of the time, which I am now convinced were erroneous.

The first case I have given somewhat in detail because the clinical history is obscure. and I believe I did not adopt the course best adapted to secure the recovery of my patient.
Case 1.-Mrs. G. C., aged 43 years, mother of four children, the youngest of whom is ten months old, was taken on Oct. 28,1890 , with severe pains in the pelvis, and I was sent for. I found that she had menstruated at the fifth, sixth and seventh month after her last delivery although still nursing her child, but for the last three months had missed her periods. During this time she had had no symptoms to induce her to believe herself pregnant, except the failure of the menses to appear. Upon making a digital examination I found the uterus somewhat enlarged. I could feel no swelling or enlargement outside of the uterus. I ordered quiet and anodyne enemata, which relieved her. On November 5, I was called in haste to see her, the husband stating that she was suffering greatly and was quite faint. I found her crying out in an agony of pain located in the pelvis, her pulse feuble, her skin cool and pale and in a condition of partial collapse. I could feel no swelling or tumor outside of the uterus but a soft fullness in Douglas' cul-de-sac. The pain, when the finger was brought in contact with the roof of the vagina, was excruciating. She vomited once or twice. The diagnosis was tubal pregnancy with rupture of the sac with hemorrhage into the pelvis. I proposed abdominal section, to which they readily consented. The hygienic surroundings were very bad, but with the aid of Dr. H. S. Brookes and two medical students, I proceeded to make an incision four inches in length. Upon opening the abdomen a quantity of blood gushed out, confirming at once the diagnosis. The sac was found connected with the right Fallopian tube. The mass was brought up and the pedicle tied with a double ligature. The tumor was then cut off. The pelvic cavity was then freely douched with hot water and Thiersch's fluid. The greatest difficulty was experienced in removing the clots, which came away in large quantities. When it was supposed that all had been removed further exploration revealed additional clots firmly adherent to the intestines, which had to be picked off with the fingers, doubt. less leaving many others. A drainage tube was placed to the bottom of Douglas' cul-de-sac and the wound closed. The woman was placed in bed in good condition and $\mathrm{Mr}$. Koch left in charge. The patient was comparatively quiet up to five o'clock next morning when he left, and having no regular nurse she was left alone. The next morning I found that she had deranged the dressings leaving the wound uncovered and the tube had been nearly forced out by her rolling and tossing about the bed; this could only be imperfectly replaced. I made an attempt to replace the tube in which I only partially succeeded. At $6 \mathrm{p}$. M. of the following day the temperature was $101 \mathrm{~F}$., pulse 143 . Later the septic symptoms became more pronounced and she died.

Case 2. Some four or five years ago I was called in consul. tation, and learned from the attending physician the following history: The woman, about 30 or 40 years of age, had missed one menstrual period. The day previous to that upon which I saw her she had gone to the basement of the house and while there was seized with a sharp, cutting pain in the pelvis accompanied by faintness. After a short time she went up stairs and was again seized with severe pain and faintness compelling her to send for her physician. He found her suffering greatly, with rapid pulse and symptoms of collapse. $\mathrm{He}$ gave her anodynes for the relief of the pain and enjoined quiet. The next day, he sent for me. The patient had rapid pulse, great prostration, abdomen distended, but with marked dull. ness in the suprapubic region. 1 diagnosed tubal pregnancy with rupture of the sac and large extravasation of blood and suggested operation. There was some delay in operating, which was done after dark and with a poor light. On opening the abdomen the blood spurted out. I fished up the ruptured sac as rapidly as I could and tied it off. I sought to get rid of the large amount of blood in the abdomen by washing it out with hot water, using eight to ten gallons for the purpose. I gave it up in despair of getting the abdomen clean, as clots continued to be found adherent to the intestines, which could oniy be removed by the fingers. Putting in a drainage tube, stitching up the wound and appiying antiseptic dressing, the patient was put to bed, hot bottles applied and other measures taken to bring about reaction, but all to no purpose, as she failed to rally and died in the night.

I received the next day a journal in which a practitioner had had a case precisely similar. Having opened the abdomen and removed the ruptured sac, he found the patient's condition such that it was necessary to terminate the operation at once if he wished to get her off the table alive. He hastily removed with his hands as much blood as he could, closed the wound and put the patient to bed, and fortunately she rallied and recovered.

I have not forgotten the lesson and have done no flushing since in these cases. 